\title{
A System for Visualizing the Process Address Space in the Context of Teaching Secure Coding in C
}

\author{
James Walker \\ Man Wang \\ jwwalker@mtu.edu \\ manw@mtu.edu \\ Michigan Technological University \\ Houghton, MI
}

\author{
Steve Carr \\ Western Michigan University \\ Kalamazoo, MI \\ steve.carr@wmich.edu
}

\author{
Jean Mayo \\ Ching-Kuang Shene \\ jmayo@mtu.edu \\ shene@mtu.edu \\ Michigan Technological University \\ Houghton, MI
}

\begin{abstract}
Seemingly small coding errors can create significant vulnerabilities in $C$ programs. This often occurs due to memory being overwritten in unexpected ways. If a student understands where program variables appear in the process address space, then she can understand the effect of writing beyond the memory allocated to a variable. With this understanding, she can tie her code to its effect within an executing process and is more likely to appreciate the significance of these seemingly harmless errors and to avoid them.

We have developed a program analysis and visualization tool to help students understand the impact of common memory errors with the goal to help students avoid introducing these errors into their code. The visualization is through the Program Address Space (PAS) window within a larger system for analysis and visualization of security issues in $\mathrm{C}$ programs. The larger system is called SecureCvisual.

In this paper, we describe our experience with teaching students fundamental concepts about process address spaces and the impact of buffer overflows using the PAS window. We also present the results from an evaluation of the tool. Our results indicate that students found the tool useful and that it enhanced the course in which it was used.
\end{abstract}

\section{CCS CONCEPTS}

\section{- Social and professional topics $\rightarrow$ Computing education.}

\section{KEYWORDS}

cybersecurity, visualization, program execution

\section{ACM Reference Format:}

James Walker, Man Wang, Steve Carr, Jean Mayo, and Ching-Kuang Shene. 2020. A System for Visualizing the Process Address Space in the Context of Teaching Secure Coding in C. In The 51st ACM Technical Symposium on Computer Science Education (SIGCSE '20), March 11-14, 2020, Portland, OR, USA. ACM, New York, NY, USA, 7 pages. https://doi.org/10.1145/3328778. 3366894

Permission to make digital or hard copies of all or part of this work for personal or classroom use is granted without fee provided that copies are not made or distributed for profit or commercial advantage and that copies bear this notice and the full citation on the first page. Copyrights for components of this work owned by others than ACM must be honored. Abstracting with credit is permitted. To copy otherwise, or republish to post on servers or to redistribute to lists, requires prior specific permission and/or a fee. Request permissions from permissions@acm.org.

SIGCSE '20, March 11-14, 2020, Portland, OR, USA

(ㄷ) 2020 Association for Computing Machinery.

ACM ISBN $978-1-4503-6793-6 / 20 / 03 \ldots \$ 15.00$

https://doi.org/10.1145/3328778.3366894

\section{INTRODUCTION}

Computer security is at the forefront of computer science. Software developers and researchers can no longer consider secure development to be a side issue since the cost of insecure software is enormous in both dollars and potential harm to businesses. As computer science educators, we have a duty to train future software professionals that security is part of the entire design and implementation process.

Despite the fact that "Improper Restriction of Operations within the Bounds of a Memory Buffer" is the number one software error in the 2019 CWE Top 25 Most Dangerous Software Errors, the problem keeps surfacing in new and old code alike. At the 2017 NSF SaTC PI meeting, Farnam Jahanian of Carnegie Mellon University stated "We don't do the easy things well and the hard things are getting harder." The SecureCvisual project is largely about teaching students to do some of the easy things well.

The SecureCvisual system leverages visualization to help students learn important concepts in secure $\mathrm{C}$ programming. It allows visualization of a program's address space, the call graph, integer conversions and the handling of sensitive data (e.g. keys and passwords). The system is comprised of two parts. An analysis system and a visualization system. The visualization takes input from the analysis system in the form of events related to program security drawn from a program execution.

This paper focuses on the the Program Address Space (PAS) component of SecureCvisual. The PAS window allows students to visualize the program's address space. Students can see how program variables are laid out in memory and how program execution changes values in memory. We evaluated the PAS window in a systems programming course at Michigan Technological University. The evaluation indicated that students found the tool useful and that it enhanced the course.

We begin the rest of this paper with an overview of related work. Then we describe the visual components of the PAS window in Section 3, our classroom use of the tool (Section 4) and give an evaluation of the use of the tool in the classroom in Section 5. Finally, we give our conclusions and future work.

\section{RELATED WORK}

For the improvement of code quality and security, a rich set of static and dynamic analysis tools have been developed. Klockwork [17], Parasoft [12], Purity [6] and Flawfinder [25] are examples. The integration of analysis tools can help developers to code defensively 
and further facilitate agile development where continuous delivery is valued.

Information visualization is also commonly applied within the field of code analysis. Astrée [1], developed by AbsInt, proves the absence of run-time errors and invalid concurrent behavior in safetycritical software written or generated in C. Results are given in graphical forms; a pie chart is available for an overview of existing problems with individual issues expandable through mouse clicks. Kiuwan [8] is a relatively new program that handles a rather comprehensive list of security problems with the support of multiple programming languages. It can be integrated into commonly used IDE platforms and generates dynamic visualization and reports. All these tools are utilities for locating security deficiencies and do not have pedagogical goals.

Visualization is an educational technique that is widely used to improve learning. There are a number of pedagogical tools for visualization of security concepts, including network protocols and defenses [7, 15, 24, 27], formal models [9, 14, 23], cryptography $[3,11,13,18-20]$ and others [2]. There are also visualization systems aimed to teach computer architecture [22,26].

Visualization tools also exist to help novice programmers learn $C[5,16,21]$. The $C$ Tutor is available online and shows the value of variables and their location in the address space of the process. Most similar to our work is SeeC [5]. This system allows execution traces to be viewed and can depict equation evaluation trees and the values of program variables, linking pointers to their target [4] The focus of these tools is different than ours in that our primary focus is teaching concepts in computer security. This means that many students using the tool have some knowledge of C. Our goal is to help them understand the execution of their code, especially in ways that can violate security. SecureCvisual can depict details like sections of the process address space, including memory addresses; it can show the assembly language listing; it can show register values and it depicts control variables on the stack. The SecureCvisual system simultaneously provides multiple security-related perspectives on the execution, including the program address space, call graph, integer conversions and the handling of sensitive data.

\section{SECURECVISUAL}

SecureCvisual depicts the execution of a student's C program. A visualization system takes input from an analysis that is run offline, using LLDB. ${ }^{1}$ The analysis generates a set of events that are processed by the visualization component. This forms an event queue and controls are provided to allow the user to determine how the events will be processed, The user can step through an execution, to replay certain parts of an execution, or create an animation at a chosen speed. The visualization system is written in Java and the system runs on any platform that support Java and LLDB.

We designed the Program Address Space visualization to reveal the contents of a process' address space as a program executes. This helps a student to make the connection between $C$ code, the contents of memory, and the effect of the execution. We believe

${ }^{1}$ We are currently switching the analysis portion over to Pin [10], using libdwarf and libelf to map addresses to variable names.

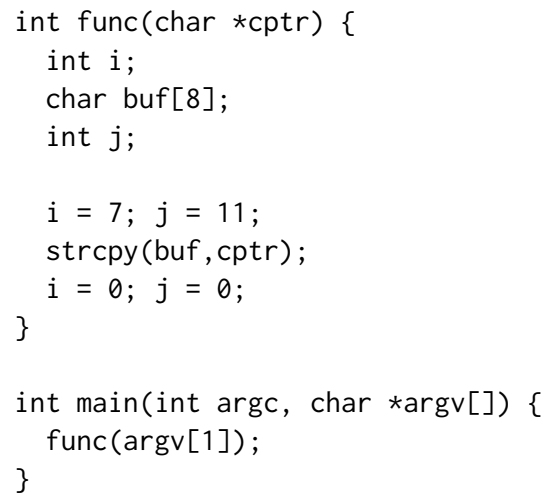

Figure 1: Example C Code: overflow.c

this connection is crucial for students to understand and avoid introducing vulnerabilities in their code.

An ability to visualize the program execution in this way facilitates instruction on many coding errors. Our primary goal was to enable students to understand: the coding practices that lead to overwriting memory with unexpected values, where an overwrite would occur, and the effect on an execution. More specifically, the visualization system can help students to understand where variables appear in the process address space; the impact of different calling conventions or compiler operation on the order in which variables appear on the stack; the implications of where a variable appears on what an attacker might achieve with an overflow; the mechanisms available to prevent an attack, e.g. a canary, as well as the coding practices that allow memory to be overwritten in unexpected ways.

\subsection{Operation}

The system is intended to be used by students with varying levels of sophistication to increase their understanding of $C$ program execution. Address space information can be displayed using different user-selected levels of detail. There are four detail modes: novice, intermediate, advanced and expert.

We discuss the information available in each of the modes provided by SecureCvisual using the $\mathrm{C}$ code in Figure 1 . This small program contains a buffer overflow vulnerability and is run using the command overflow ABCDEFG12345678.

3.1.1 Novice Mode. At the novice level, the PAS window shows information about a program's variables, including the address, name, type, size, value and to where the variable points (if anywhere). In addition, the command line used to invoke the program is given.

Figure 2 shows the contents of the PAS window in novice mode for our example code. The PAS is available through the Program Address Space tab. The Call Graph, File Operations, and Sensitive Data tabs access other visualizations that are consistent with the their names. The Source Code tab is discussed later in this section. As can be seen in the figure, the variables for the function main are displayed. The <address width> size reminds students that pointer variables have the same size and that the size is related to the address size of the machine. 


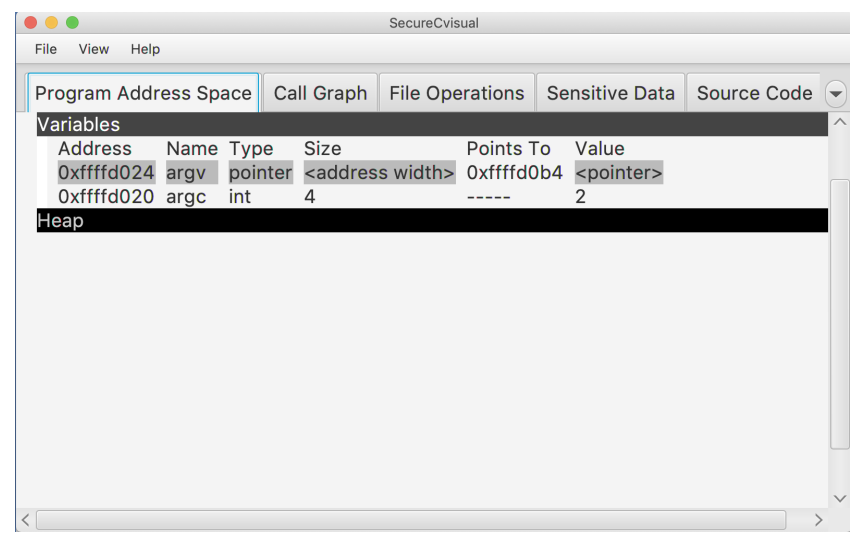

Figure 2: PAS Window for Novice Mode

In addition to the PAS window, SecureCvisual shows the $\mathrm{C}$ source through the Source Code tab. The next line to be executed is orange. Figure 3 has an example of the source code window for the example code with the next line to be executed at func (argv[1]); .

\begin{tabular}{|c|c|c|c|c|c|}
\hline-0 & & SecureCvisual & & & \\
\hline File View Help & & & & & \\
\hline Program Address Space & Call Graph & File Operations & Sensitive Data & Source Code & - \\
\hline $\begin{array}{l}1 \text { \#include <string.h> } \\
2 \\
3 \text { void func(char *cptr) }\{ \\
4 \text { int } i ; \\
5 \text { char buf }[8] ; \\
6 \text { int } j ; \\
7 \\
8 \quad i=7 ; \\
9 \quad j=11 ; \\
10 \quad \text { strcpy(buf,cptr); } \\
11 \quad i=0 ; \\
12 \quad j=0 ; \\
13\} \\
14 \text { int main (int argc, char * } \\
15 \text { func(argv }[1]) ; \\
16\}\end{array}$ & $\operatorname{argv}[])\{$ & & & & \\
\hline
\end{tabular}

Figure 3: Source Code Window for Novice Mode

Finally, the user can step through events using the buttons in the Playback window (Figure 4). The user can step forward or backward at the granularity of one source code line (one "event"). The user can also play through program events at various speeds, or skip to the beginning or end of the program execution.

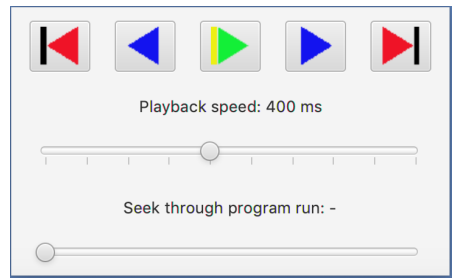

Figure 4: Playback Window

Novice mode is for the beginning $\mathrm{C}$ programmers and does not differentiate where variables are stored within the address space. It also does not automatically give a mapping from source to assembly. The variables section shows only variables that are in scope. The novice mode intends to help students get a better understanding of scope and pointers. A more advanced view of the program is available in the other modes as discussed next.

3.1.2 Intermediate Mode. Intermediate mode introduces visualization for the CPU registers, program sections and stack. Consider Figure 5; SecureCvisual shows three sections (code, regular and data) with information about where they are located and the permissions the program has on those sections. Additionally, the stack frame for main is shown with the parameters argc and argv, the return address and the dynamic link (the previous value of \%ebp).

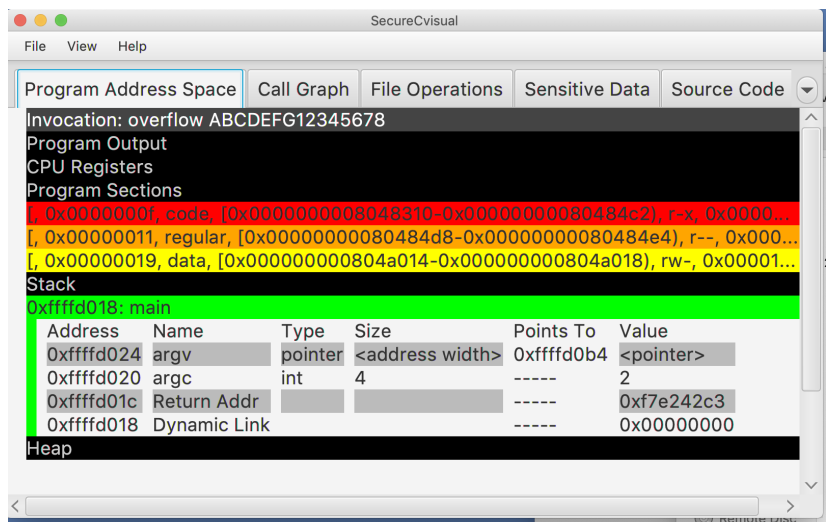

Figure 5: PAS for Intermediate Mode

Figure 6 shows the stack after executing the line $i=7 ; j=11$; in the function func. (The depicted view was obtained by scrolling past the invocation and other information). The stack frames for both main and func are shown with all parameters and local variables.

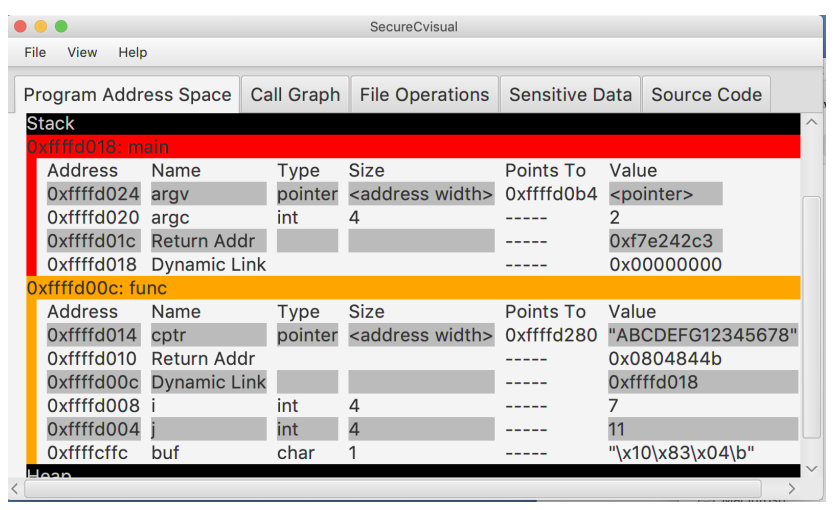

Figure 6: Stack Upon Entry to func

Notice that the next line to be executed will overflow the buffer buf via the call to strcpy (). Hence, Figure 6 shows the stack before the overflow.

When the user steps past the next line, she sees the effect, as shown in Figure 7. Notice that the values of both $i$ and $j$ have 


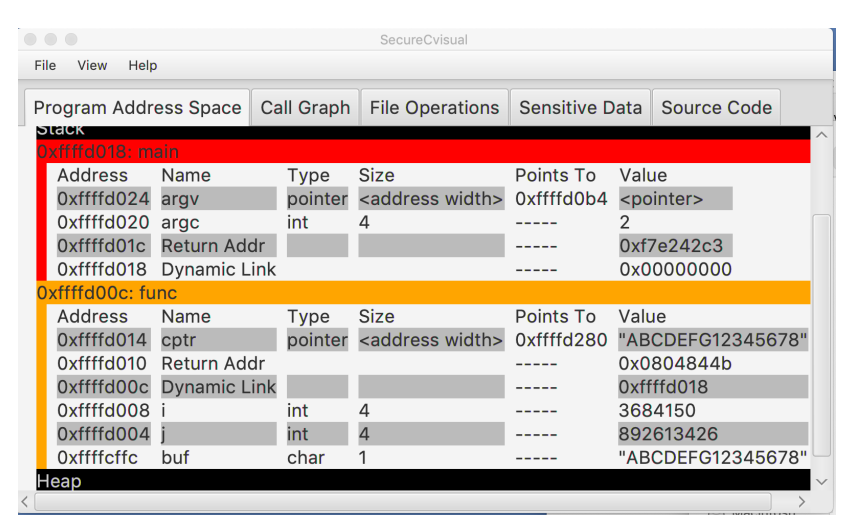

Figure 7: Stack After Buffer Overflow

changed due to the overflow. Currently, we do not monitor library calls, so execution steps past the call to strcpy atomically.

In addition to the visualization of the stack, program sections and CPU registers, the intermediate mode automatically shows the assembler code along with the $\mathrm{C}$ source code. It provides the ability to see the assembler corresponding to a particular line of $\mathrm{C}$ code (the source code executed). This allows a student to see the $\mathrm{C}$ source, the corresponding assembler instructions, and the execution all at the same time, as well as to move forward and backward through the code execution.

3.1.3 Other modes. Advanced mode allows the user to see the path to main, including libc_start, on the stack. Expert mode shows all program sections contained in the executable. These modes allow more advanced users to see all the parts of their program when loaded into memory.

\section{CLASSROOM USE}

We used the system in a junior-level course on systems programming. This is a required course for CS majors in our department. Students in this course have had our introductory programming course that uses Java, a course in $\mathrm{C}$ and assembly language programming, and a course in computer organization. The systems programming course covers file $\mathrm{I} / \mathrm{O}$, process creation and management, linking and libraries, interprocess communication, and socket programming, all through the Linux system call interface. We have identified this course for inclusion of certain topics in computer security including input filtering, integer errors, and buffer overflows. The course has been in our curriculum for more than ten years.

The visualization system was used both for lecture and for student assignments. We discuss its use for student assignments in detail in Section 5. Lectures in the class commonly are rooted in code examples. We used the system to demonstrate execution of this code where it was beneficial to the lecture.

Subjectively, use of the tool seemed to stimulate questions more than code examples that were discussed without a demonstration or that were demonstrated using a debugger. The ability to use automatically generated depictions of the address space allowed multiple examples to be shown in class without generating new overheads, generating depictions on the blackboard, or explaining elements of the address space without a corresponding depiction.
The ability to step forward and backward through an execution allowed certain steps of the execution to be easily revisited and discussed in detail in response to student questions, easily tying the code to its effect on the execution.

In the next section, we discuss an evaluation of the tool we conducted in this course.

\section{EVALUATION}

\subsection{Environment, Procedure and Goals}

We conducted an evaluation of the PAS window in a junior-level Systems Programming course with a total of 65 students. Students were given a pre-test on their knowledge prior to any lectures.

Then lectures were given on the contents of the process address space, including activation records. The PAS window was used during the lecture to depict the address space and execution for example programs, to demonstrate the effect of a buffer overflow, and to explore the use of a canary as a mechanism for buffer overflow detection.

Students were then given an extra credit assignment in which they used the PAS window, in intermediate mode, to solve questions over the lecture material. After the assignment, the students evaluated their experience with the PAS window, and their understanding of the material was further assessed through a post-test in the form of a midterm exam. The course emphasis is on programming; there are no homework assignments. Students are given review problems prior to the midterm, then the review problem solutions are discussed in class before the midterm.

We collected 59 valid pre-tests, 61 valid post-tests, and 31 valid evaluation forms. (Thirty-one students turned in the extra-credit assignment.)

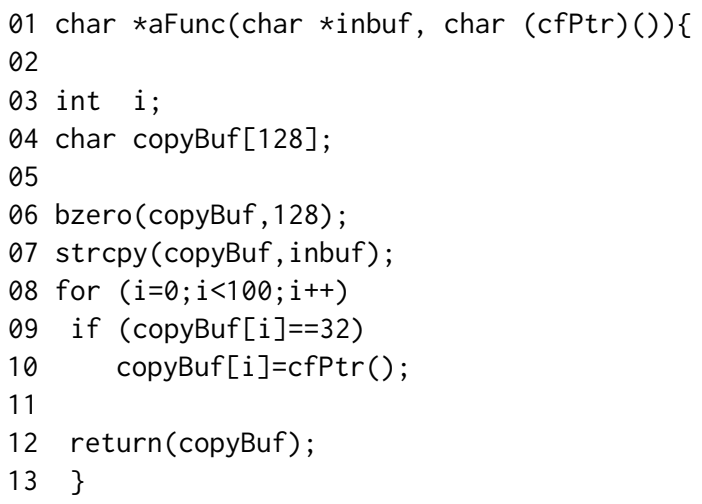

Figure 8: Code for Test Question

The pre-test and the post-test each included three questions that were essentially the same across the two tests. The questions were over the code of Figure 8. Question Q1 required students to identify where the memory for copyBuf would appear in the process' address space. Q2 required students to recreate the activation record on entry to the procedure. It required that students specify both the set of variables (excluding temporaries) and the order according to assumptions that were explained in class and consistent throughout the class discussions. This question was worth ten points. Finally, 
Q3 asked students evaluate modification of the code in Figure 8 by inserting the code in Figure 9. ${ }^{2}$ The question asked, "Suppose that the variable canary is deeper in the stack (at a higher address) than both $i$ and copyBuf. Does this stack canary protect the return address from being overwritten by the call to strcpy at line 7?" Students were instructed to explain their answer. The question was worth five points.

\section{At line 2: int canary $=37$;}

At line 11: if (canary!=37) exit (0);

Figure 9: Canary Code

\subsection{Test Performance}

Table 1 and Figure 10 have the mean, standard deviation and confidence intervals $\left(\mathrm{CI}^{-}\right.$and $\left.\mathrm{CI}^{+}\right)$of each question in the pre-test and post-test. The correctness of all questions improved in the post-test. Q1, Q2 and Q3 showed a 66\%, 58\% and 57\% increase in correctness, respectively. Q2 was the most challenging question. It had the lowest rate of correctness in the both pre- and post-test. A common mistake was for students to include the correct set of variables, but to depict them in the incorrect order. In the responses to Q3, many students did not recognize that the canary variable could be overwritten with the expected value.

Table 1: The Means $(\mu)$, Standard Deviations $(\sigma)$ and $95 \%$ Confidence Intervals of the Pre- and Post-test Questions

\begin{tabular}{|c|c|c|c||c|c|c|}
\hline & \multicolumn{3}{|c||}{ Pre-test } & \multicolumn{3}{c|}{ Post-test } \\
\hline & $\mathrm{Q} 1$ & $\mathrm{Q} 2$ & $\mathrm{Q} 3$ & $\mathrm{Q} 1$ & $\mathrm{Q} 2$ & $\mathrm{Q} 3$ \\
\hline$\mu$ & 0.08 & 0.00 & 0.02 & 0.74 & 0.58 & 0.59 \\
\hline$\sigma$ & 0.28 & 0.00 & 0.13 & 0.45 & 0.50 & 0.50 \\
\hline$C I^{-}$ & 0.01 & 0.00 & 0.00 & 0.62 & 0.45 & 0.46 \\
\hline$C I^{+}$ & 0.16 & 0.00 & 0.05 & 0.85 & 0.70 & 0.71 \\
\hline
\end{tabular}

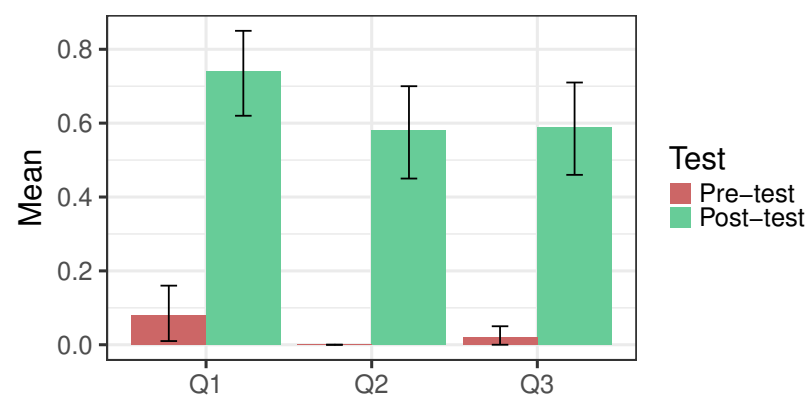

Figure 10: The Means with 95\% Confidence Intervals of the Pre- and Post-test Questions

${ }^{2}$ The variable canary should be declared volatile to ensure clang does not optimize out the canary. This level of understanding is not required in our course.
We further investigate whether students' performance between the pre-test and post-test had a significant improvement. The parametric method ANOVA was employed as the main tool with the non-parametric Kruskal-Wallis (KW) test used for the confirmation of the result, both at the $95 \%$ significance level. The $p$-values of the ANOVA test were $2.20 \times 10^{-16}, 2.69 \times 10^{-13}$, and $9.79 \times 10^{-13}$ in Q1, Q2 and Q3, all of which are less than 0.05. The KW test showed the same result. Combined with their increased means from the pre-test to the post-test (Table 1), this indicates that the performance on all questions had significantly improved.

Students performed very well on the extra credit. The average score was 13.75 out of 15 . The assignment required students be able to identify where program variables would appear in the process address space, a detailed depiction of a stack frame, and analysis of the impact of an overflow on the value of local variables. We did not perform a direct comparison between the test performance of students who did the extra credit and those that did not.

The results for Q2 and Q3 are lower than we believe we can achieve. We anticipate that using the tool in assignments required by all students will allow us to address the ordering misconceptions that surfaced in Q2. The last question, Q3, is a little more challenging because it requires a student to incorporate their understanding of the stack contents and additionally consider the flexibility an attacker has for crafting an overflow string to reach various end goals. We believe that a deeper understanding of ordering of variables on the stack will help students to have a better grasp of concepts tested by Q3. Importantly, the tool provides the ability for a virtually limitless set of examples, constructed by an instructor or a student, to address virtually any misconception that might arise.

Table 2: PAS Window Rating and Usage Questions

\begin{tabular}{|c|l|}
\hline \multicolumn{2}{|c|}{ Rating Questions } \\
\hline Q1 & $\begin{array}{l}\text { The PAS helped me to see how components of a process } \\
\text { address space are laid out in memory }\end{array}$ \\
\hline Q2 & $\begin{array}{l}\text { The PAS helped me understand where the memory for local, } \\
\text { global, and static variables is allocated }\end{array}$ \\
\hline Q3 & The PAS helped me understand activation records \\
\hline Q4 & $\begin{array}{l}\text { The PAS helped me to see how a buffer overflow can change } \\
\text { values of variables adjacent to the buffer that was overflowed }\end{array}$ \\
\hline Q5 & $\begin{array}{l}\text { I understand process execution better after using the PAS } \\
\text { Q6 }\end{array} \begin{array}{l}\text { I was able to identify concepts I did not understand after } \\
\text { using the software }\end{array}$ \\
\hline Q7 & $\begin{array}{l}\text { The PAS window enhanced the course in the area of program } \\
\text { execution }\end{array}$ \\
\hline Q8 & The text of the PAS window was easy to read \\
\hline Q9 & It was easy to identify one stack frame from another \\
\hline Q10 & It was easy to identify the contents of each stack frame \\
\hline Q11 & $\begin{array}{l}\text { It was easy to identify the address and value of variables in } \\
\text { the data section }\end{array}$ \\
\hline
\end{tabular}




\subsection{Evaluation by Students}

We used a set of questions (Table 2) to collect information on student perception of the effectiveness of the tool. We also gathered information on the time spent using the PAS window. The first 11 rating questions evaluated the effectiveness of the PAS window. The choices were: 1:strongly disagree, 2:disagree, 3:neutral, 4:agree, and 5:strongly agree. Q12 to Q14 evaluated the time participants spent on the tool. The choices for Q12 were 1:less than 5 mins, 2:5-9 mins, 3:10-14 mins, 4:15-30 mins, and 5:more than 30 mins. The choices for Q13 are 1:once, 2:twice, 3:3-4 times, 4:5-10 times, and 5:more than 10 times. The choices for Q14 were 1:less than 5 mins, 2:5-14 mins, 3:15-29 mins, 4:30-60 mins, and 5:more than 1 hour.

Table 3: The Means $(\mu)$, Standard Deviations $(\sigma)$, and Confidence Intervals of Evaluation Questions

\begin{tabular}{|c|c|c|c|c|c|c|c|}
\hline & Q1 & Q2 & Q3 & Q4 & Q5 & Q6 & Q7 \\
\hline$\mu$ & 4.23 & 4.19 & 3.58 & 4.29 & 4.00 & 3.77 & 4.03 \\
\hline$\sigma$ & 0.72 & 0.70 & 1.03 & 0.86 & 0.68 & 0.73 & 0.60 \\
\hline$C I^{-}$ & 3.97 & 3.95 & 3.22 & 3.99 & 3.76 & 3.51 & 3.82 \\
\hline$C I^{+}$ & 4.48 & 4.44 & 3.94 & 4.59 & 4.24 & 4.03 & 4.25 \\
\hline & Q8 & Q9 & Q10 & Q11 & Q12 & Q13 & Q14 \\
\hline$\mu$ & 3.81 & 4.16 & 4.23 & 4.32 & 2.55 & 1.97 & 3.65 \\
\hline$\sigma$ & 0.91 & 0.97 & 0.76 & 0.83 & 1.06 & 0.98 & 0.95 \\
\hline$C I^{-}$ & 4.13 & 4.50 & 4.49 & 4.62 & 2.92 & 2.31 & 3.98 \\
\hline$C I^{+}$ & 3.49 & 3.82 & 3.96 & 4.03 & 2.18 & 1.62 & 3.31 \\
\hline
\end{tabular}

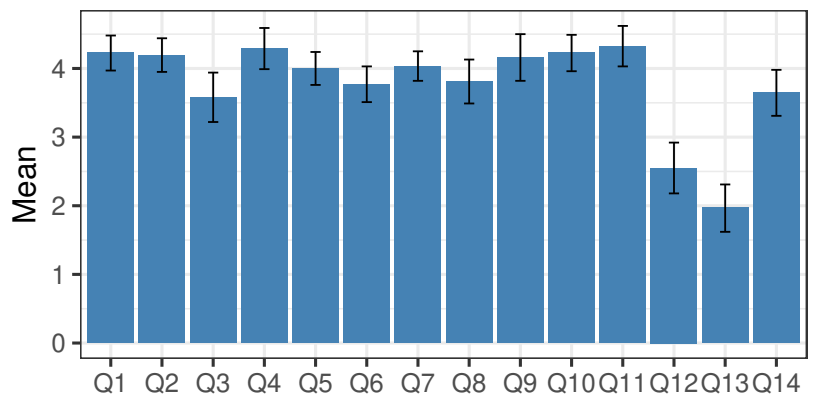

Figure 11: The Means with Confidence Intervals of Evaluation Rating and Usage Questions

Table 3 lists the mean, standard deviation and confidence intervals for each of the evaluation questions. Figure 11 depicts the means and confidence intervals for both the evaluation and usage questions.

The rating of the tool was positive with a mean of 4.06 across questions. Q11 received the highest rating of 4.32, which means students believed that the PAS window made it easy to identify the address and value of variables in the data section. Q3, which is associated with improving students' understanding of activation records, had the lowest rating of 3.58. As indicated in students' comments in the write-in questions, it is believed that the tool generally helped students understand activation records. However, the interface freezing and text scaling issues detailed in their writein comments might have been the reason why this question received the lowest rating.

The means of Q12, Q13 and Q14 are 2.55, 1.97 and 3.65. This indicates that students generally used the PAS Window twice and 15 to 30 minutes in total. It usually took students $10-15$ minutes to understand the process address space and execution using the PAS window.

The four write-in questions were used to collect information on the students' major, their comments on the use of the PAS window to explore program execution, features to add, and problems with installation. The feedback on the PAS functionality and the visualization was generally positive. Some students stated that "The $P A S$ window offers a helpful and informative glimpse into how a program's address space might look and how it's organized. I learned and felt more confident about PAS concepts after using the tool", "I enjoy having a visual aid for programs and how things work in memory when writing programs", and "it clearly helped me to understand the process address spaces". Students also mentioned some problems with the user interface. The tendency to freeze when resizing the window and a scaling problem of the text font were reported.

Most of the students did not encounter any problem during installation. One student mentioned that the program would not start the first time, possibly due to an improper Java version. As for the features to add, the majority of students found the available features satisfying. Some comments were also given to help further improvement. A student suggested that "it would be helpful to list the size of the data types". Some students indicated that "the assembly code window could have been bigger for better readability" and "the tutorial should be updated". We will incorporate the suggestions in our future versions of the program.

\section{CONCLUSIONS AND FUTURE WORK}

Lecturing with the PAS window of SecureCvisual is straightforward. We currently use examples that were created prior to lecture. We plan to support real-time example development and modification.

A paired pre- and post-test showed that students' knowledge about the contents of the address space created for a particular program was significantly increased when the tool was used for lecture and extra-credit assignments. The students' evaluation was generally positive feedback, with a mean of 4.06 out of 5 across all questions; students believed the PAS window helped them to understand process address spaces and enhanced the course. Suggestions for further improvement were mainly on the user interface. We will address the comments appropriately in a future version.

We plan to test the PAS window in classes at different levels. We are also testing the other components of SecureCvisual. An Eclipse module is under development that provides s student feedback on secure coding practices while she edits her code.

The most recent version of SecureCvisual system is available at vaccs.cs.mtu.edu.

\section{ACKNOWLEDGMENTS}

This work was supported in part by the National Science Foundation under grants DGE-1522883, DGE-1523017 and DUE-1245310. 


\section{REFERENCES}

[1] AbsInt. [n. d.]. Astrée. https://www.absint.com/astree/index.htm. Accessed: 2019-02-14.

[2] James W. Benham. 1992. A Geometric Approach to Presenting Computer Representations of Integers. SIGCSE Bull. 24, 4 (Dec. 1992), 27-28.

[3] Derek Ebeling and Rob Santos. 2007. Public Key Infrastructure Visualization. 7. Comput. Sci. Coll. 23, 1 (Oct. 2007), 247-254.

[4] Matthew Egan and Chris McDonald. [n. d.]. SeeC Viewing Execution Traces. https://seec-team.github.io/seec/seec-view.html Accessed: 2019-11-25.

[5] Matthew Heinsen Egan and Chris McDonald. 2014. Program Visualization and Explanation for Novice C Programmers. In Proceedings of the Sixteenth Australasian Computing Education Conference - Volume 148 (ACE '14). Australian Computer Society, Inc., Darlinghurst, Australia, Australia, 51-57. http: $/ /$ dl.acm.org/citation.cfm?id=2667490.2667496

[6] IBM. [n. d.]. Rational Purify. https://www-01.ibm.com/software/awdtools/purify/ compare.html. Accessed: 2019-02-14.

[7] Niakam Kazemi and Shiva Azadegan. 2010. IPsecLite: A Tool for Teaching Security Concepts. In Proceedings of the 41st ACM Technical Symposium on Computer Science Education (SIGCSE '10). ACM, New York, NY, USA, 138-142.

[8] Kiuwan. [n. d.]. Kiuwan. https://www.kiuwan.com/. Accessed: 2019-02-14.

[9] Yifei Li, Steve Carr, Jean Mayo, Ching-Kuang Shene, and Chaoli Wang. 2012. DTEvisual: A Visualization System for Teaching Access Control Using Domain Type Enforcement. Fournal of Computing Science in College 28, 1 (October 2012), 125-132.

[10] C. K. Luk, R. Cohn, R. Muth, H. Patil, A. Klauser, G. Lowney, S. Wallace, V. J. Reddi, and K. Hazelwood. 2005. Pin: Building Customized Program Analysis Tools with Dynamic Instrumentation. In Proceedings of 2005 Conference on Programming Language Design and Implementation (PLDI). Chicago, Illinois.

[11] Jun Ma, Jun Tao, Melissa Keranen, Jean Mayo, Ching-Kuang Shene, and Chaoli Wang. 2014. SHAvisual: A Secure Hash Algorithm Visualization Tool. In Proceedings of the 2014 conference on Innovation \& technology in computer science education. ACM, 338-338.

[12] Parasoft. [n. d.]. Parasoft. https://www.parasoft.com/. Accessed: 2019-02-14.

[13] Dino Schweitzer and Leemon C. Baird III. 2006. The design and use of interactive visualization applets for teaching ciphers. In Proceedings of the 7th Annual IEEE Information Assurance Workshop. 69-75.

[14] Dino Schweitzer, Mike Collins, and Leemon C Baird III. 2007. A visual approach to teaching formal models in security. In Proceedings of the 11th Colloquium for Information Systems Security Education (CISSE). 69-75.
[15] Dino L. Schweitzer, Leemon C. Baird III, Mike D. Collins, Wayne C. Brown, and Mike Sherman. 2006. GRASP: A visualization tool for teaching security protocols. In Proceedings of the 10th Colloquium for Information Systems Security Education. 75-81.

[16] P. A. Smith and G. I. Webb. 1995. Transparency Debugging with Explanations for Novice Programmers. In Proceedings of the Second International Workshop on Automated and Algorithmic Debugging (AADEBUG'95), M. Ducass (Ed.). IRISACNRS.

[17] Rogue Wave Software. [n. d.]. Klocwork. https://www.roguewave.com/productsservices/klocwork. Accessed: 2019-02-14.

[18] Jun Tao, Jun Ma, Melissa Keranan, Jean Mayo, and Ching-Kuang Shene. 2012. ECvisual: A Visualization Tool for Elliptic Curve Based Ciphers. In roceedings of the 43rd ACM technical symposium on Computer Science Education. ACM, 571-576.

[19] Jun Tao, Jun Ma, Melissa Keranen, Jean Mayo, and Ching-Kuang Shene. 2011. DESvisual: A Visualization Tool for the DES Cipher. Fournal of Computing Science in College 27, 1 (October 2011), 81-89.

[20] Jun Tao, Jun Ma, Melissa Keranen, Jean Mayo, Ching-Kuang Shene, and Chaoli Wang. 2014. RSAvisual: A Visualization Tool for the RSA Cipher. In Proceedings of the 45th ACM technical symposium on Computer science education. ACM, 635-640.

[21] Minoru Terada. 2005. ETV: A Program Trace Player for Students. In Proceedings of the 10th Annual SIGCSE Conference on Innovation and Technology in Computer Science Education (ITiCSE '05). ACM, New York, NY, USA, 118-122. https://doi. org/10.1145/1067445.1067480

[22] Kenneth Vollmar and Pete Sanderson. 2006. MARS: An Education-oriented MIPS Assembly Language Simulator. In Proceedings of the 37th SIGCSE Technical Symposium on Computer Science Education (SIGCSE '06). ACM, 239-243.

[23] Man Wang, Steve Carr, Jean Mayo, Ching-Kuang Shene, and Chaoli Wang. 2014. MLSvisual: A Visualization Tool for Teaching Access Control Using Multi-Level Security. In Proceedings of the 2014 conference on Innovation \& technology in computer science education. ACM, 93-98.

[24] Justin Warner, David Musielewicz, G. Parks Masters, Taylor Verett, Robert Winchester, and Steven Fulton. 2010. Network Firewall Visualization in the Classroom. 7. Comput. Sci. Coll. 26, 2 (Dec. 2010), 88-96.

[25] David A. Wheeler. [n. d.]. Flawfinder. https://dwheeler.com/flawfinder/ Accessed: 2019-02-14.

[26] Cecile Yehezkel, Mordechai Ben-Ari, and Tommy Dreyfus. 2005. Computer Architecture and Mental Models. In Proceedings of the 36th SIGCSE Technical Symposium on Computer Science Education (SIGCSE '05). ACM, 101-105.

[27] Xiaohong Yuan, Percy Vega, Yaseen Qadah, Ricky Archer, Huiming Yu, and Jinsheng Xu. 2010. Visualization Tools for Teaching Computer Security. Trans. Comput. Educ. 9, 4 (Jan. 2010), 20:1-20:28. 